\title{
Successful Haploidentical Transplantation with High Doses of CD34+-Selected Peripheral Blood Stem Cells in Wiskott-Aldrich Syndrome: A Case Report
}

\author{
Kurekci AE ${ }^{1 *}$, Eker $\mathbf{I}^{2}$, Atay $\mathrm{AA}^{3}$ and Gürsel $\mathrm{O}^{4}$ \\ ${ }^{1}$ Department of Pediatric Hematology, Lösante Hospital, \\ Ankara, Turkey \\ ${ }^{2}$ Department of Pediatric Hematology-Oncology, Afyon \\ Kocatepe University, Turkey \\ ${ }^{3}$ Department of Pediatric Hematology-Oncology, İstanbul \\ Yeni Yüzyll University, Turkey \\ ${ }^{4}$ Department of Pediatric Hematology-Oncology, Sağllk \\ Bakanlığı University, Turkey \\ *Corresponding author: Kurekci AE, Department \\ of Pediatric Hematology, Lösante Hospital, Kızılcaşar \\ Mah. 23 Nisan Caddesi 2705. Sokak No: 20 İncek, 06830 \\ Ankara, Turkey
}

Received: June 21, 2017; Accepted: July 28, 2017; Published: August 28, 2017

\begin{abstract}
Wiskott-Aldrich syndrome (WAS) is a rare X-linked recessive, primary immunodeficiency, characterized by eczema, microthrombocytopenia, recurrent infection, and increased susceptibility to autoimmune diseases and lymphoreticular malignancies. To date, HSCT is the only curative therapy. HSCT using HLA-matched related or unrelated donors is highly successful in treating WAS, but these donors may not always be available. We describe a 20 months-old-boy with WAS, who was transplanted by using highly-purified, $16.11 \times 106 / \mathrm{kg} \mathrm{CD}^{+} 4^{+}$stem cells from his HLA 2-loci mismatched mother, because of lacking donor and suffering life threatening, ongoing sinopulmonary infections. Conditioning consisted of busulfan $(16 \mathrm{mg} / \mathrm{kg})$, cyclophosphamide $(200 \mathrm{mg} / \mathrm{kg})$, rabbit anti-thymocyte globulin $(10 \mathrm{mg} / \mathrm{kg})$ and fludarabine $(160$ $\mathrm{mg} / \mathrm{m}^{2}$ ). He received methylprednisolone for graft-versus-host disease (GvHD) prophylaxis. The time for neutrophil, platelet and erythrocyte recovery was 11 days, 14 days and 19 days, respectively. He showed immunologic reconstitution by day +180 . He developed grade II aGVHD on day +12 , and grade I cGVHD on day +240 , resolution achieved in aGVHD with i.v. methylprednisolone and cyclosporine, in CGVHD with oral prednisolone. He is well and alive up to now six years after transplantation. He didn't have any serious infection. Fluorescence in situ hybridization analysis revealed sustained full donor-type engraftment and flow cytometric analysis revealed sustained immunologic reconstitution. The convenient results in this patient can encourage further investigation of the role of using 'high doses' of related haploidentical CD34-selected peripheral blood stem cells in patients with WAS at early stages of disease, before the beginning of severe, life-threatening complications.
\end{abstract}

Keywords: Wiskott-Aldrich syndrome; Haploidentical blood stem cell transplantation; High dose CD $34^{+}$cells

\section{Introduction}

In 1937, Wiskott described a clinical entity characterized by thrombocytopenia, eczema, bloody diarrhea, and recurrent otitis media in male infants [1]. After rediscovery in 1954 by Aldrich as an X-linked recessive disorder, it was designated the Wiskott-Aldrich syndrome (WAS) [2]. X-linked thrombocytopenia (XLT), sometimes associated with mild eczema and/or infections, was recognized in the 1960s and was suspected to be a variant of WAS. This was confirmed when patients with XLT were shown to have mutations in the Wiskott-Aldrich syndrome protein gene (WAS). WAS mutations result in 3 distinct clinical phenotypes: classic WAS, XLT, and $\mathrm{X}$-linked neutropenia, and a strong genotype phenotype correlation has been suggested. Mutations completely averting WAS protein (WASP) expression typically lead to the classic phenotype [3]. The classic WAS phenotype with microthrombocytopenia, severe eczema, increased susceptibility to pyogenic and opportunistic infections, and increased risk of autoimmune disease and cancer usually leads to death in early childhood or adolescence if left untreated.

Curative treatment by allogeneic hematopoietic stem cell transplantation (HSCT) should be offered to all such patients.
The outcome is excellent if performed early in life from a human leukocyte antigen-matched related or unrelated donor [4]. The results of mismatched related or unrelated donor transplantations are associated with an increased risk of graft rejection and graft-versushost disease (GVHD) [5]. To overcome these limitations, a 'high dose' of highly purified CD34 ${ }^{+}$peripheral blood stem cells (PBSC) from his HLA 2-loci mismatched mother was used in this patient. This report details and draws attention to this kind of haploidentical peripheral blood stem cell transplantation in a patient with severe WAS.

\section{Case Presentation}

A four-month-old male infant was brought to our clinic with a history of itchy, scaly lesions on his neck and scalp, epistaxis and recurrent pulmonary infections since one month of age. He was the first child of non-consanguineous parents, born at term following an uncomplicated pregnancy. On examination, he was active and well hydrated. His weight, height and head circumference was within normal limits and his vital parameters were stable. There was no pallor, mucocutaneous bleeds or ear discharge. The dry and scaly lesions on his neck and scalp were consistent with eczema. Examination of other systems did not reveal any abnormality. Whole 
blood count showed a hemoglobin concentration of $9.4 \mathrm{~g} / \mathrm{dl}$, platelet count of $16000 / \mu \mathrm{L}$, total leukocyte count of $8800 / \mu \mathrm{L}, \mathrm{MCV} 74.7 \mathrm{fL}$ and MPV 5.4 fL. A blood smear revealed \%12 neutrophils (ANC: 1056/ $\mu \mathrm{L}), 62 \%$ lymphocytes $(5456 / \mu \mathrm{L}), 12 \%$ monocytes, $14 \%$ eosinophils $(1232 / \mu \mathrm{L})$. There were also seen very small platelets $(50 \%$ of normal size) on the blood smear, which was consistent with MPV value but there weren't any big or giant platelets. Bone marrow aspirate smear findings were normal in terms of the presence, maturation, and differentiation of megakaryocytes, erythroid or granulocytic precursors. Wiskott-Aldrich syndrome was suspected in the patient because of microthrombocytopenia, recurrent infections and eczema. Molecular genetic analysis revealed WAS mutation. Liver and kidney functions, serum immunoglobulin levels, and urinalysis were all normal. The diagnosis was confirmed as severe WAS (score 5) by clinical findings and molecular genetic analysis.

Because of lacking related or unrelated matched donor and because of ongoing, recurrent, severe sinopulmonary and middle ear infections, he underwent haploidentical HSCT from his mother at 20 months of age. He received a myeloablative regimen consisting of busulfan (16 mg/kg), cyclophosphamide $(200 \mathrm{mg} / \mathrm{kg})$, rabbit antithymocyte globulin $(10 \mathrm{mg} / \mathrm{kg})$ and fludarabine $\left(160 \mathrm{mg} / \mathrm{m}^{2}\right)$. He also received methylprednisolone for graft-versus-host disease (GvHD) prophylaxis. $\mathrm{T}$ cell-depletion and positive selection of peripheral CD $34^{+}$stem cells from his G-CSF stimulated mother was performed using the method of immunomagnetic separation (CliniMACS, Miltenyi Biotec). The number of infused CD $34^{+}$cells was 16.11x106/ $\mathrm{kg}$. He achieved full hematopoietic engraftment with the time for neutrophil, platelet, and erythrocyte recovery being 11 days, 14 days, and 19 days, respectively. He developed grade II aGVHD on day +12 , and grade I cGVHD on day +240 , resolution achieved in aGVHD with i.v. methylprednisolone and cyclosporine A, in cGVHD with oral prednisolone. He was positive for cytomegalovirus which was detected by PCR in his blood, urine and stool samples on day +6 and resolved with ganciclovir. He is now 61/2 age, he has not experienced any serious infection and fluorescence-in-situ-hybridization analysis revealed sustained full donor-type chimerism and flow cytometric analysis revealed immunologic reconstitution.

\section{Conclusion}

Hematopoietic stem cell transplantation offers successful results for patients with WAS. Without HSCT 50\% of these patients will die from infection, lymphoproliferative disease or autoimmune diseases by the third decade of their life. The WAS mutation affects hematopoietic and lymphoid compartments; both of these compartments are corrected by HSCT. To facilitate multilineage donor cell engraftment, most patients are conditioned with both immunosuppression and high dose chemotherapy. There is an almost 2 -fold reduction in mortality among patients with full-chimerism compared to those with mixed or split chimerism. This showed the importance of achieving full donor chimerism in these patients who underwent HSCT. Mixed chimerism also is associated with a significantly higher risk for developing autoimmune manifestations after HSCT [6].

HSCT using HLA-identical sibling donors is highly successful in treating WAS, with approximately $88 \%$ event-free survival. The most commonly used conditioning regimen is the combination of busulfan
$(1 \mathrm{mg} / \mathrm{kg} \times 16$ doses) and cyclophosphamide $(200 \mathrm{mg} / \mathrm{kg}$ total dose), with or without anti-thymocyte globulin. In younger patients, busulfan is metabolized faster. Therefore, it is prudent to monitor levels and target the dose to achieve a steady state concentration of greater than $200 \mathrm{ng} / \mathrm{ml}$ to assure engraftment. It is determined that patient age, disease severity, and splenectomy did not affect outcome in HSCT of WAS patients in which HLA-identical sibling marrow grafts were used $[7,8]$.

Results of alternative donor HSCT for WAS having improved over time, particularly for unrelated marrow grafts. Most studies have shown approximately $70 \%-78 \%$ long-term survival. The International Bone Marrow Transplant Registry (IBMTR) which was facilitated in 2001 reported that, age of patients who are 5 years or older was associated with an increased risk of mortality after HSCT [6]. Survival for patients younger than 5 years was about the same as HLA-identical sibling HSCTs. In addition, it is found by several studies that there are comparable survival rates among recipients of HLA-matched related and unrelated HSCTs. The most commonly used regimen was a combination of busulfan, cyclophosphamide, and ATG. It is suggested that targeting the dose of busulfan to achieve a steady state concentration above $400 \mathrm{ng} / \mathrm{ml}$ facilitates engraftment.

Encouraging results have also been reported for umbilical cord blood transplants, but most of them are case reports. Among 15 patients reported by Ozsahin, et al. event-free survival was approximately $70 \%$. It was similar to recipients of unrelated marrow grafts. Most patients in their reports were conditioned with the combination of busulfan, cyclophosphamide and antithymocyte globulin, which they found to be an important factor for improved survival when compared to other regimens. Reconstitution of immunity after cord blood approximates that observed with unrelated marrow grafts [9].

Although the historical results are less encouraging, there is more information about haploidentical transplants for WAS than umbilical cord blood transplants. John MJ, et al. reported literature review of eight studies between 1979 and 2014 about haploidentical transplants for WAS a case report about un-manipulated haploidentical transplant in WAS [10]. According to their review, between 1979 and 2014, there are 17 reported haploidentical transplants in the age group of 2-12 years with 8 survivors. Conditioning regimens used were cytosine arabinoside/total body irradiation (TBI) (2/17), busulfan/cyclophosphamide (5/17), cyclophosphamide/ TBI (3/17) and fludarabine based (4/17). Stem cell source was bone marrow in majority (13/17) and the rest used peripheral blood stem cell products $(4 / 17)$. T cell depletion (TCD) was performed in most of the cases (12/17). Apart from their case, one more unmanipulated haplo-identical transplant was reported. There is a 4-5-fold increase in mortality after haploidentical compared to HLA-identical sibling grafts $[7,11]$. These reports show a high incidence of graft failure and poor immune reconstitution following $\mathrm{T}$ cell-depleted haploidentical HSCTs. Schwinger, et al. provided a promising case report of "megadose" purified $\mathrm{CD}_{3} 4^{+}$haploidentical grafts used to overcome the barrier to engraftment without endangering GVHD [12]. Our convenient results in this patient in a way that supports the results of Schwinger, et al. can encourage further investigation of the role of using 'high doses' of related haploidentical CD34-selected peripheral blood stem cells in patients with WAS at early stages of disease before the beginning of severe, life-threatening complications. 


\section{References}

1. Wiskott A. Familiaereer, angeborner morbus Werlhoffi? Monatsschr Kinderheilkd. 1937; 68: 212-216.

2. Aldrich RA, Steinberg AG, Campbell DC. Pedigree demonstrating a sexlinked recessive condition characterized by draining ears, eczematoid dermatitis and bloody diarrhea. Pediatrics. 1954; 13:133-138.

3. Albert MH, Bittner TC, Nonoyama S, Notarangelo LD, Burns S, Imai K et al. X-linked thrombocytopenia (XLT) due to WAS mutations: clinical characteristics, long-term outcome, and treatment options. Blood. 2010; 115 3231-3238.

4. Ochs HD, Filipovich AH, Veys P, Cowan MJ, Kapoor N. Wiskott-Aldrich syndrome: diagnosis, clinical and laboratory manifestations, and treatment. Biol Blood Marrow Transplant. 2009; 15: 84-90.

5. Brochstein JA, Gillio AP, Ruggiero M, Kernan NA, Emanuel D, Laver J, et al. Marrow transplantation from human leukocyte antigen-identical or haploidentical donors for correction of Wiskott-Aldrich syndrome. J Pediatr. 1991; 119: 907-912.

6. Burroughs L, Woolfrey A. Hematopoietic cell transplantation for treatment of primary immune deficiencies. Cell Ther Transplant. 2010; 2.

7. Filipovich $\mathrm{AH}$, Stone JV, Tomany SC, Ireland M, Kollman C, Pelz CJ, et al. Impact of donor type on outcome of bone marrow transplantation for WiskottAldrich syndrome: collaborative study of the International Bone Marrow Transplant Registry and the National Marrow Donor Program. Blood. 2001; 97: 1598-1603.
8. Slattery JT, Sanders JE, Buckner CD, Schaffer RL, Lambert KW, Langer FP, et al. Graft-rejection and toxicity following bone marrow transplantation in relation to busulfan pharmacokinetics. Bone Marrow Transplant. 1995; 16: $31-42$.

9. Ozsahin H, Le Deist F, Benkerrou M, Cavazzana-Calvo M, et al. Bone marrow transplantation in 26 patients with Wiskott-Aldrich syndrome from a single center. J Pediatr. 1996; 129: 238-244.

10. John MJ, Philip CC, Mathew A, Williams A, Kakkar N. Un-manipulated Haploidentical Transplant in Wiskott-Aldrich Syndrome. Indian Pediatr. 2017 54: 327-328.

11. Fischer A, Landais P, Friedrich W, Gerritsen B, Fasth A, Porta F, Vellod $A$, et al. Bone marrow transplantation (BMT) in Europe for primary immunodeficiencies other than severe combined immunodeficiency: a report from the European Group for BMT and the European Group for Immunodeficiency. Blood. 1994; 83: 1149-1154.

12. Schwinger W, Urban C, Lackner H, Kerbl R, Benesch M, Dornbusch HJ, et al. Unrelated partially matched peripheral blood stem cell transplantation with highly purified $\mathrm{CD} 34^{+}$cells in a child with Wiskott-Aldrich syndrome. Bone Marrow Transplant. 2000; 26: 235-237.
Ann Hematol Oncol - Volume 4 Issue 8 - 2017

ISSN : 2375-7965 | www.austinpublishing group.com

Kurekci et al. () All rights are reserved
Citation: Kurekci AE, Eker I, Atay AA and Gürsel O. Successful Haploidentical Transplantation with High Doses of CD34+-Selected Peripheral Blood Stem Cells in Wiskott-Aldrich Syndrome: A Case Report. Ann Hematol Oncol. 2017; 4(8): 1167 